\title{
Statistical aspects of tip vortex cavitation inception and desinence in a nuclei deplete flow
}

\author{
M.T. Khoo · J.A. Venning - B.W. Pearce - P.A. Brandner
}

\begin{abstract}
Tip vortex cavitation (TVC) inception and desinence behaviour of a NACA 0012 cross-section, elliptical hydrofoil is investigated from a statistical perspective in a cavitation tunnel. Measurements were made for incidences from $4^{\circ}$ to $16^{\circ}$ and Reynolds numbers from $1.0 \times 10^{6}$ to $2.1 \times 10^{6}$. The statistics of TVC inception were quantified by taking repeated measurements of the time until the appearance of a tip vortex cavity for a range of fixed incidences. In other experiments, the angle of attack was continuously increased until inception then decreased until desinence for a range of fixed cavitation numbers. The data were primarily acquired via an automated process using a laser and photodiode to detect the presence of a cavity. Measurements show that TVC inception in a nuclei deplete flow is a probabilistic process for which a large dataset is required for accurate characterisation. The probability of ingesting and activating a nucleus increases with time at a given test condition due to the increased volume of water exposed to low pressures. TVC desinence exhibits far less statistical variation than inception and is largely independent of the natural nuclei population. It does, however, exhibit hysteresis which is dependent on the topology of the cavitating flow. For the desinence of unattached cavitation, there is a small hysteresis between the inception and desinence indices. However, desinence is delayed for attached cavitation.
\end{abstract}

Keywords Hydrodynamics · Tip vortex cavitation · Nucleation · Cavitation

M.T. Khoo

Maritime Division

Defence Science and Technology Group

Melbourne, Australia

E-mail: matthew.khoo@dst.defence.gov.au

M.T. Khoo · J.A. Venning · B.W. Pearce · P.A. Brandner

Cavitation Research Laboratory

University of Tasmania, Launceston, Australia 


\section{Graphic abstract}

Typical natural nuclei population (left) and notional capture of a nucleus into a trailing vortex (right)

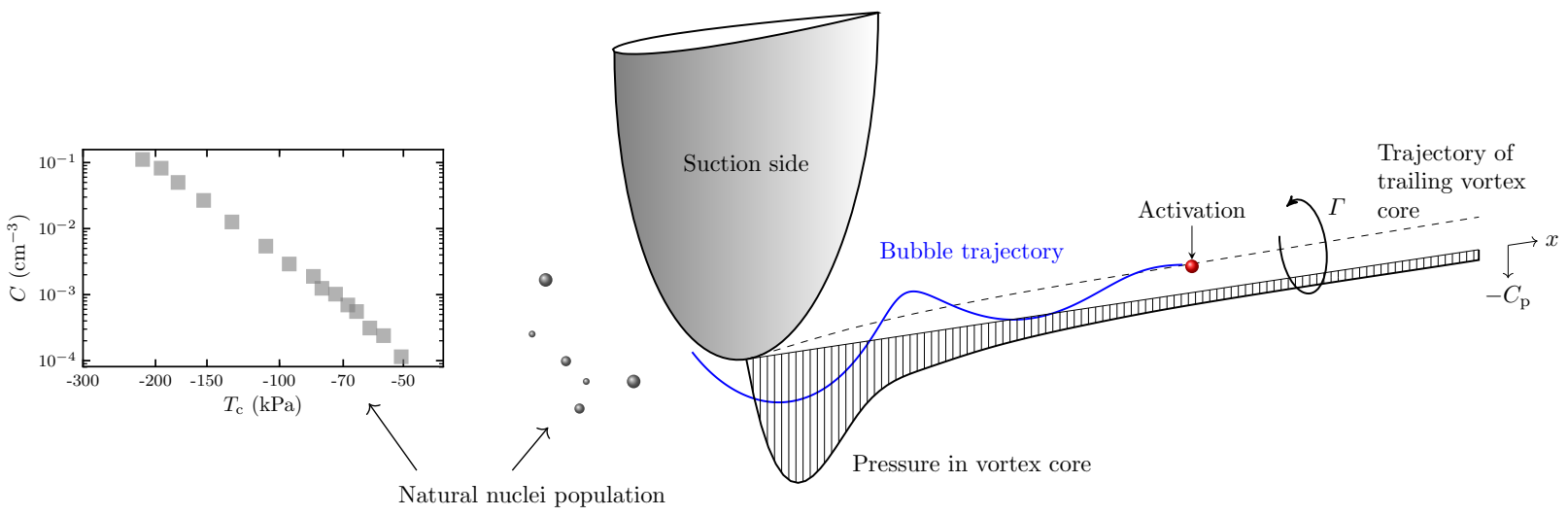

Variation of time until inception with incidence (left) and statistical distribution of 455 measurements at $6.0^{\circ}$ (right)

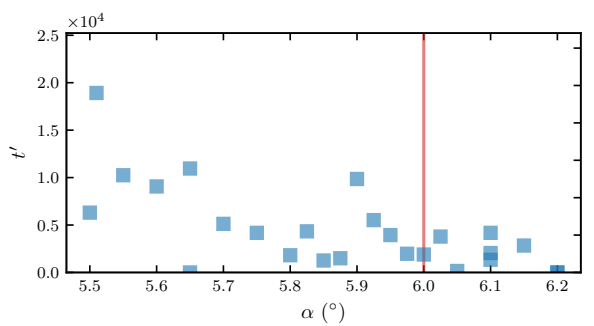

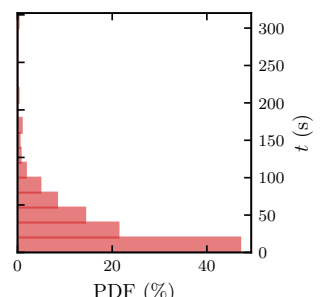

PDF (\%)
Cavitation desinence hysteresis due to cavity topology

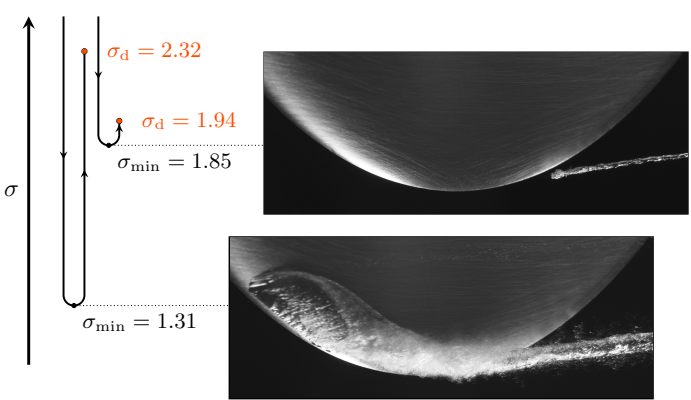




\section{Introduction}

Trailing vortices are inherently generated from the tips of three-dimensional lifting surfaces. Low pressures exist in these regions of concentrated vorticity (Fig. 1), making the flow susceptible to tip vortex cavitation (TVC) once the pressure reduces below vapour pressure. However, water is capable of withstanding high tensions (Blake Jr, 1949), such that cavitation does not incept at vapour pressure. The effective strength of the water is dependent on the size, concentration and spatial distribution of nuclei, such as microbubbles, in the volume. The size of these inhomogeneities, their distance from the vortex, and the flow field pressure gradients determine which nuclei are ingested into a vortex and how quickly. If a nucleus is drawn towards the low-pressure vortex core (Fig. 1) and exposed to its size-dependent critical pressure, its equilibrium becomes unstable and it grows rapidly, filling with vapour. The tension in the vortex varies in the streamwise direction, decreasing as the vortex diffuses downstream of the lifting surface. As the pressure increases, the cavity can no longer be sustained and it collapses. TVC is generally undesirable as it can be detrimental to the hydrodynamic, acoustic and structural performance of marine propellers and control surfaces, as well as turbomachinery.

Numerous TVC studies have been carried out in test facilities since the mid-twentieth century. In a seminal study by McCormick (1962), the critical cavitation index for desinence (the disappearance of cavitation) about a hydrofoil was found to depend on Reynolds number and incidence. A power-law fit applied to these data yields a Reynolds number power index of about 0.4, which can also be derived from boundary layer and airfoil theory (Billet and Holl, 1979; Franc and Michel, 2006). This index has been widely used for TVC scaling studies (Arndt and Dugue, 1992; Fruman and Dugue, 1994; Briançon-Marjollet and Merle, 1996). However, upon closer inspection of McCormick's data, the index decreases with increasing incidence $\left(0.4\right.$ for $4^{\circ}, 0.3$ for $8^{\circ}$ ) for a rectangular-planform hydrofoil. Changes in incidence alter flow topology, in particular the boundary layer condition, which will in turn affect the vortex core size and minimum pressure. Therefore, the scaling of cavitation behaviour from model to full-scale is presumably only accurate for the flow topology studied and is not universally applicable. Moreover, several studies have indicated that the index varies with Reynolds number (Amromin, 2006; Shen et al., 2009), which suggests that further work is required to understand viscous effects on TVC.

In addition to flow topology, water quality, i.e. free and dissolved gas content, has also been shown to influence cavitation inception behaviour. McCormick (1962) measured the effect of dissolved air content on the critical cavitation index and deduced that undissolved gas influences TVC desinence behaviour. This has been confirmed in subsequent studies, in which the cavitation inception indices of propellers and hydrofoils were ob-

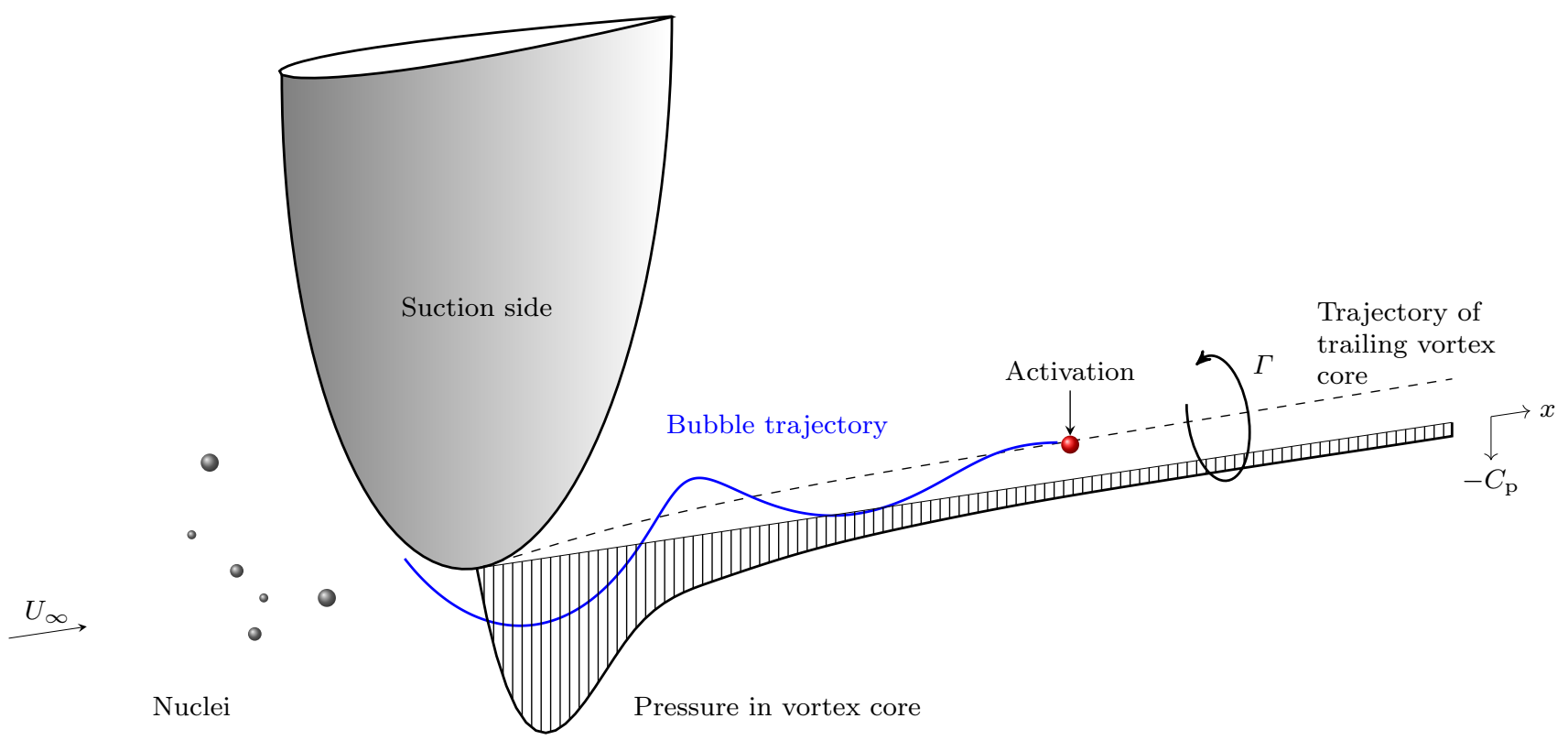

Fig. 1 Notional capture of a nucleus (red sphere) into the trailing vortex of a lifting surface. The blue line denotes the nucleus trajectory. The freestream velocity is $U_{\infty}$ and the circulation is $\Gamma$. The generic variation of the core pressure coefficient, $C_{\mathrm{p}}$, with streamwise location, $x$, is also shown with the minimum pressure occurring close to the tip and pressure recovery downstream as the vortex diffuses. 
served to scale or increase with injected nuclei populations (Briançon-Marjollet and Merle, 1996; Gindroz and Billet, 1998; Song et al., 2017). McCormick (1962) also noted the lower repeatability of cavitation inception measurements compared to desinence, and attributed this to the random distribution of nuclei in the flow which plays a role in the inception process. While this makes desinence behaviour easier to study scientifically, inception is likely to be of more interest for practical applications.

Extensive measurements of nuclei in facility and environmental waters have been carried out since the midtwentieth century using optical, acoustic and hydrodynamic techniques (Gavrilov, 1969; O'Hern et al., 1988; Shen et al., 1984; Gindroz, 1995; Randolph et al., 2014). Hydrodynamic measurements typically report lower populations, partly because optical techniques may also detect non-cavitating particles and also due to the quality of the water itself. However, the power-law trends observed across all measurements are generally comparable (Khoo et al., 2020) with a phenomenological model proposed by Franklin (1992) which describes the relationship between nuclei size and concentration as a power law with an index of -4 . While this indicates a universal characteristic of water, Khoo et al. (2020) noted temporal variations in the nuclei population which naturally occurs in a cavitation tunnel, also known as the natural nuclei population, which may be described as a nuclei deplete population. This is in contrast to those populations found in cavitating wakes or flows deliberately injected with microbubbles, whose nuclei concentrations are several orders of magnitude higher, as discussed by Brandner (2018) and Khoo et al. (2020). Temporal variations in natural nuclei populations in cavitation tunnels highlight the importance of understanding nuclei population dynamics and of monitoring. This is a necessary consideration for TVC experiments in nuclei deplete flows.

Water tensile strength describes the ability of a body of water to withstand pressures below vapour pressure without cavitating. Venning et al. (2018) showed that for 'infinite' sampling times, water has no strength due to the presence of large nuclei, albeit in low concentrations. However, for finite durations, there is general agreement that water tensile strength is statistical in nature due to the spatial distribution and temporal variation of nuclei in the flow (Arndt and Keller, 1992; Briançon-Marjollet and Merle, 1996; Mørch, 2000). Different definitions of water tensile strength can be found in TVC inception studies, with some using an event rate approach to account for the randomness of the nuclei distribution (Gindroz, 1995; Briançon-Marjollet and Merle, 1996), and others making multiple water tensile strength measurements at each test condition and considering the strength of the weakest nuclei found to be representative of the strength of the water (Arndt and Keller, 1992). Arndt and Maines (1994) extrapolated the relationship between test section pressure and velocity at inception to zero velocity to determine a critical pressure at inception. These definitions of tension have been applied to TVC inception scaling laws to account for nuclei effects, providing reasonable correlations (Arndt and Dugue, 1992; Arndt and Maines, 1994; Briançon-Marjollet and Merle, 1996). Given these considerations, TVC inception should be studied from a statistical perspective, with previous studies having done this with varying degrees of fidelity.

TVC inception is influenced not only by the random distribution of nuclei in the flow, but also by nuclei or bubble dynamics, which determine their motion and growth in a pressure field. A bubble typically follows a spiral trajectory as it is drawn towards the vortex core by the radial pressure gradient. Drag, lift and added mass forces serve to retard the motion of the bubble, affecting the number of orbits the bubble performs prior to capture in the core (Oweis et al., 2005). It has been found that larger bubbles have shorter capture times in vortical flows as the force induced by the radial pressure gradient increases with $R^{3}$, where $R$ is the bubble radius (Oweis et al., 2005). Zhang et al. (2017) suggested that a critical nucleus size for inception exists, defined as the largest bubble with a capture time less than a critical capture time (dependent on hydrofoil size and flow velocity). Therefore, larger, weaker bubbles may be present in the flow but will not be activated if they are in sufficiently low concentrations, passing the location of minimum pressure without ingestion into the vortex core. This work has provided the basis for the development of a TVC prediction model (Chen et al., 2019). In addition, pressure fluctuations in the vortex (Green, 1991; Arndt and Maines, 1994; Sinding et al., 2018) and vortex core wandering (Baker et al., 1974; Pennings et al., 2015) are statistical in nature and further complicate the process of nuclei capture and activation. It is evident that bubble dynamics and behaviour of the vortical flow play an important role in TVC inception.

Only a limited number of TVC inception studies have been conducted in a statistical manner. Statistical models have been used to characterise nuclei distributions (Messino et al., 1963) which were coupled with computational fluid dynamics and bubble dynamics models to predict TVC event rates for hydrofoils and propellers (Hsiao and Chahine, 2005, 2008). The majority of TVC inception studies are based on small sample sizes, and hence do not adequately address the statistical nature of inception. Six or more TVC inception 
and water tensile strength measurements were made at each test condition by Arndt and Keller (1992), but this is a relatively small sample size in the context of statistical investigations. Several studies on travelling bubble cavitation about headforms using an event rate approach have been carried out. Schiebe (1969) calculated a probable cavitation occurrence rate based on measured nuclei distributions and compared it with experimental measurements of cavitation event rates with mixed success, citing a lack of accuracy in the nuclei measurement and recommending the use of larger statistical samples. Meyer et al. (1992) used Monte Carlo simulations to model the random processes in an experiment and while there was good agreement with experimental results, the lack of a quantitative definition of inception made it difficult to be sure about the validity of the results. Ceccio and Brennen (1991) measured several thousand cavitation events about headforms and successfully correlated these with measured nuclei distributions. While some statistical studies of cavitation inception exist, they are concerned with other types of cavitation than TVC and have produced mixed results.

TVC inception is an inherently statistical process for which the availability of detailed statistical studies is limited. The present work seeks to gain insight into the probabilistic nature of TVC inception by using larger datasets to more accurately characterise the phenomenon compared to event rate approaches used in past studies. This will contribute to the improvement of TVC prediction and management capabilities for practical applications.

\section{Experimental overview}

\subsection{Test facility}

Hydrofoil TVC measurements were made in the cavitation tunnel at the Australian Maritime College Cavitation Research Laboratory (AMC CRL). A schematic of the tunnel is shown in Fig. 2. The variable-pressure, closed-circuit water tunnel has a volume of $365 \mathrm{~m}^{3}$. The test section has a $0.6 \mathrm{~m} \times 0.6 \mathrm{~m}$ cross section and is $2.6 \mathrm{~m}$ in length. The working fluid is demineralised water. The test section centreline pressure can be varied between $4-400 \mathrm{kPa}$ and the nominal flow velocity range is $2-12 \mathrm{~m} / \mathrm{s}$.

The nuclei (free gas) and dissolved gas contents in the working fluid can be controlled in the tunnel. This enables reliable and repeatable cavitation experiments to be performed. A large downstream tank allows bubbles to be removed from the bulk flow via gravity separation. The resorber in the lower limb of the tunnel circuit promotes bubble dissolution by means of long

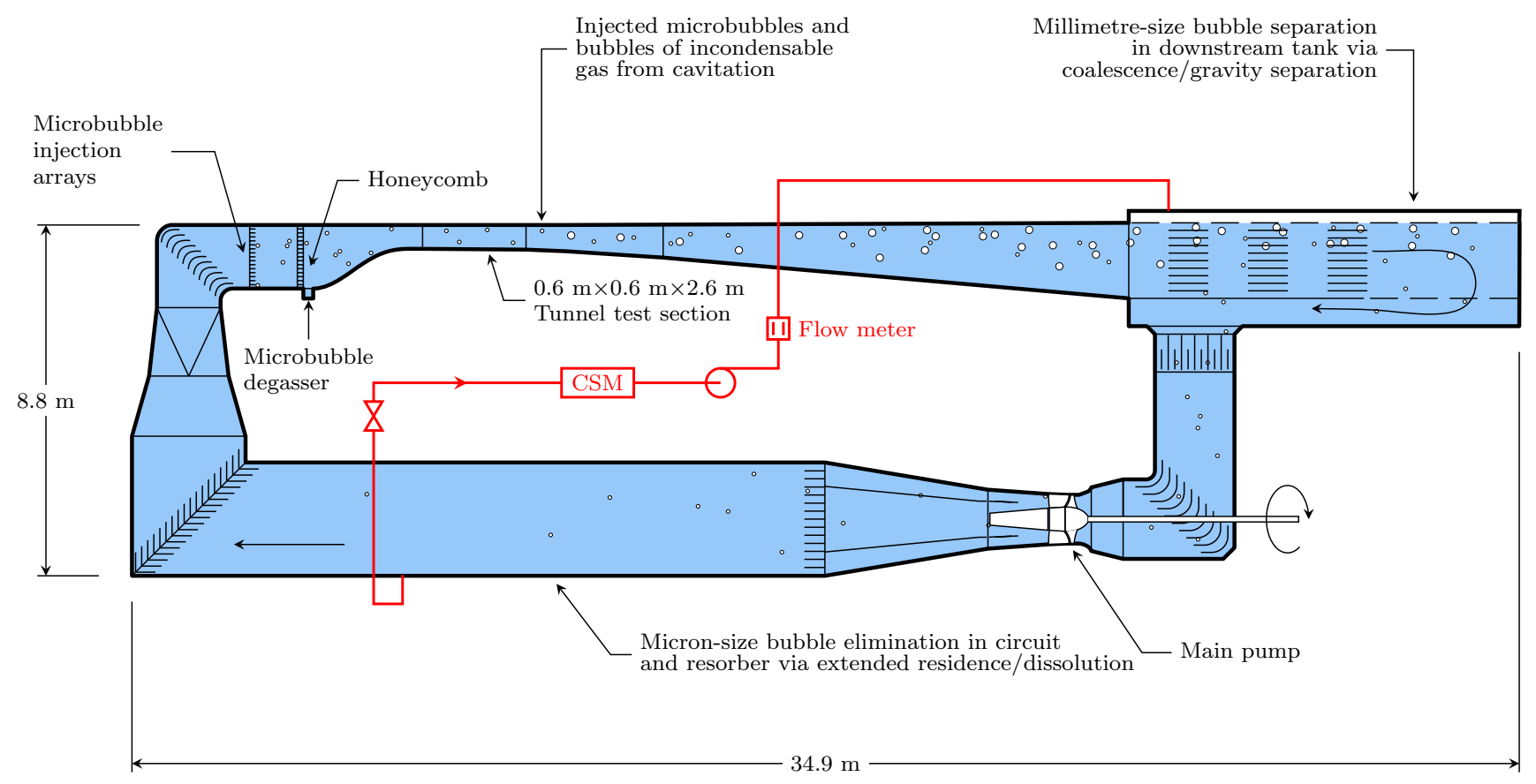

Fig. 2 Schematic of the AMC cavitation tunnel. The circuit architecture allows for continuous removal of microbubbles or large volumes of injected incondensable gas. Ancillaries for microbubble seeding and for degassing of water are shown. Microbubbles may be either injected for modelling cavitation nucleation or generated by the cavitation itself. For the present study, water was sampled from the lower-limb resorber through the cavitation susceptibility meter (CSM) for natural nuclei population measurements. 
exposure to high static pressure. A microbubble degassing system is used to lower the dissolved oxygen content of the water via the injection of bubbles into the test section at low pressures and velocities. Dissolved gas molecules are drawn out of solution, resulting in the growth of the injected bubbles which are removed in the downstream tank. A microbubble injection system enables the free gas content to be controlled during cavitation studies. An ever-present natural nuclei population has been shown to exist in the tunnel water, regardless of whether microbubble injection is used (Khoo et al., 2020). Further details on this test facility can be found in Brandner et al. (2007).

\subsection{Nuclei and dissolved gas contents}

The natural nuclei population in the tunnel water was used for this study. No additional nuclei were injected into the flow. The population was monitored using a cavitation susceptibility meter (CSM). This is a hydrodynamic measurement technique whereby water is sampled from the circuit and passed through a centrebody venturi. The water is subjected to a tension in the venturi and a nucleus will cavitate if exposed to a tension which exceeds its size-dependent critical pressure, $p_{\mathrm{c}}$. Cavitation events are counted using a piezoceramic sensor and an event rate is determined. A nuclei distribution can be characterised by conducting a tension sweep, where each tension has a corresponding cumulative nuclei concentration. Further details on the CSM operation can be found in Khoo et al. (2016).

A typical natural nuclei distribution in the AMC cavitation tunnel is provided in Fig. 3. The cumulative concentration increases as the tension applied to the water in the venturi increases (i.e. with increasing magnitude of tension, $T_{\mathrm{c}}=p_{\mathrm{c}}-p_{\mathrm{v}}$, where $p_{\mathrm{v}}$ is vapour pressure). The cumulative distribution in Fig. 3 is consistent with previous measurements for which a power-law trend was observed (Venning et al., 2018; Khoo et al., 2020).

The dissolved oxygen content was maintained between 2.6 and $5.0 \mathrm{mg} / \mathrm{L}$ (i.e. $28 \%-61 \%$ relative to satu-

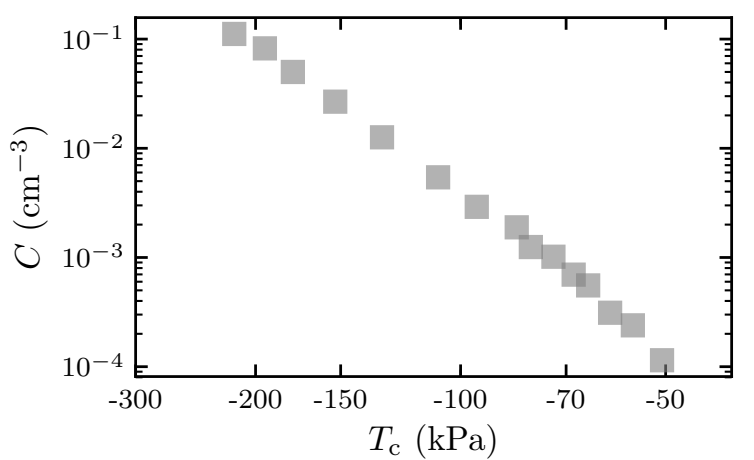

Fig. 3 A typical natural nuclei population in the AMC cavitation tunnel measured using a cavitation susceptibility meter. Nuclei concentration, $C$, is plotted against the critical pressure of a nucleus, $T_{\mathrm{c}}$. The distribution follows a powerlaw trend.

ration at atmospheric pressure) throughout the experiment. The relative saturation level of dissolved oxygen in the water, $r_{\text {up }}$, using the plenum upstream of the test section as the reference location, was maintained between $12 \%-83 \%$ during the present study which ensured that the natural nuclei population remained at the baseline, as described in Khoo et al. (2020). The definition of this relative saturation parameter and rationale for its use are provided in Khoo et al. (2020). 2.3 Experimental setup

A schematic of the experimental setup is given in Fig. 4. A stainless steel, elliptical planform, NACA 0012 hydrofoil with a $150 \mathrm{~mm}$ root chord length and an aspect ratio of 1.5 was used for this study. It was mounted to a force balance with integrated angular encoder and stepper motor, allowing for computer-controlled angular positioning and a rate of change of about $0.14^{\circ} / \mathrm{s}$. The assembly was installed at the test section mid-span in the ceiling. The mid-chord of the hydrofoil was $1.3 \mathrm{~m}$ from the test section entrance and was also the axis of rotation.

A laser and photodiode setup similar to that used by Yakushiji (2009) was the primary method for the detection of the presence of a tip vortex cavity. The laser

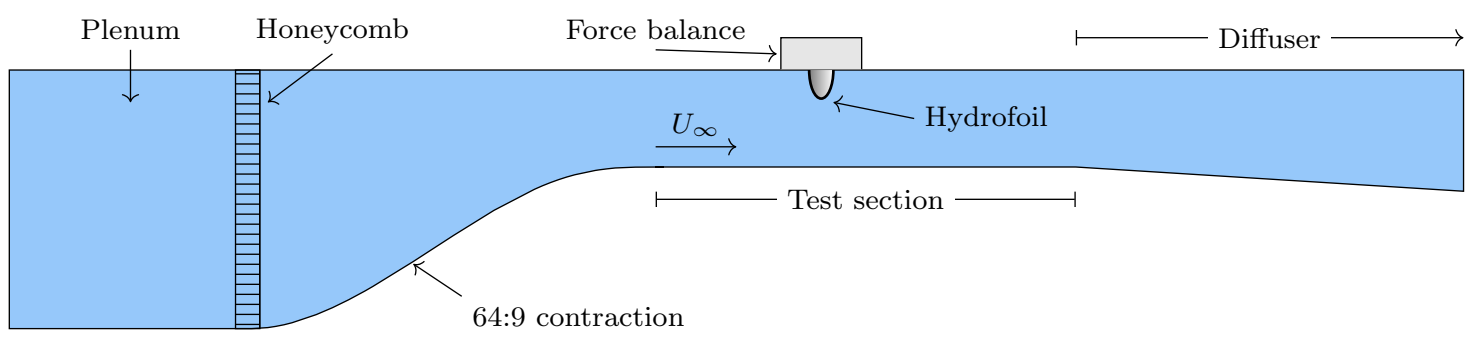

Fig. 4 Schematic of the experimental setup in the upper limb of the AMC cavitation tunnel, showing the hydrofoil located within the test section. The force balance allows for angular positioning of the hydrofoil. The test section velocity is $U_{\infty}$. 
was located on one side of the test section and aimed about $0.15 c$ downstream of the hydrofoil tip, where $c$ is the root chord length. This position was determined in part by prior observations of the inception location, and in part by optical access constraints. The photodiode was positioned on the opposite side of the test section. The appearance of a tip vortex cavity in the laser beam scatters the light and reduces the intensity detected by the photodiode. TVC inception and desinence were indicated by a decrease and increase in the diode output voltage, respectively. Note that this method cannot be used to detect cavities downstream of the laser beam. Arndt and Maines (2000) commented that inception can occur further downstream from the tip in 'strong' water (nuclei deplete flows), however, the present study is concerned with cavitation inception behaviour in the tip region.

High resolution photographs were taken of different cavity topologies in the vicinity of the hydrofoil tip using a digital single-lens reflex camera and stroboscopic lighting.

Two main methodologies were used to investigate TVC behaviour. Either the incidence or the cavitation number was varied. The Reynolds number $\left(R e=\frac{U_{\infty} c}{\nu}\right.$, where $\nu$ is the fluid kinematic viscosity) and cavitation number $\left(\sigma=\frac{p_{\infty}-p_{\mathrm{v}}}{\frac{1}{2} \rho U_{\infty}^{2}}\right.$, where $p_{\infty}$ is the freestream pressure in the test section at the same height as the hydrofoil tip and $\rho$ is the fluid density) were maintained as constant where possible. The water temperature remained between $17.4^{\circ} \mathrm{C}$ and $28.2^{\circ} \mathrm{C}$ for the data presented in this paper.

\section{Results and discussion}

A series of different experiments were performed to investigate different aspects of tip vortex cavitation inception in nuclei deplete flows. As such, the definition of cavitation inception varied slightly with test methodology. The definition associated with each dataset is provided in the following discussion.

\subsection{Statistics of cavitation inception and desinence}

The variation of time until inception for a range of incidences at fixed Reynolds and cavitation numbers is shown in Fig. 5. The incidence was continuously increased from a non-cavitating condition at $5.0^{\circ}$ to a prescribed incidence between $5.5^{\circ}$ to $6.2^{\circ}$, at which point timing began. The time until TVC inception, as detected by the photodiode, was recorded and the test was repeated 25 times across the range of incidences. A time of zero was recorded if inception occurred prior to reaching the prescribed incidence. Measurements were repeated at $6.0^{\circ}$, producing a large data set of 455 points from which the statistical distribution of TVC inception can be quantified. Time has been non-dimensionalised by the time scale $U_{\infty} / c$, which enables comparisons with flows at different scales. If the nuclei population is appropriately scaled, then for the same dimensionless time, the rate of nuclei capture and activation is equivalent regardless of model size, flow velocity and pressure field (Lecoffre, 1999).

The time until inception tends to decrease with increasing incidence. This is not unexpected given that
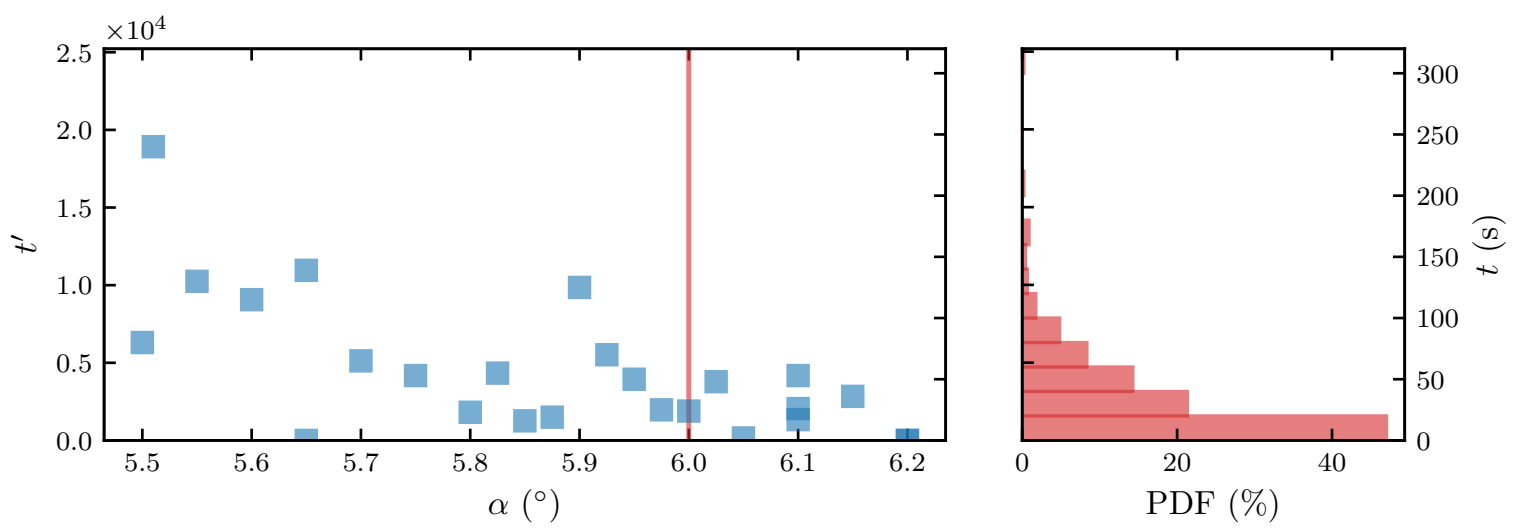

Fig. 5 Variation of time until inception across a range of incidences, $\alpha$ (blue squares, left). Dimensional, $t$, and non-dimensional, $t^{\prime}=t U_{\infty} / c$, time are shown, where $U_{\infty}$ is the flow velocity and $c$ is the maximum chord length. Inception is detected when a cavity breaks a laser beam about $0.15 \mathrm{c}$ downstream of the tip. Hydrofoil incidence was changed from $5.0^{\circ}$ (non-cavitating state) to the prescribed value, at which point timing began. The vertical scatter represents the random nature of nuclei ingestion. The cavity generally incepted sooner at higher incidences. The vertical, red line denotes the incidence $\left(6.0^{\circ}\right)$ at which 455 repeated measurements were made. The corresponding probability distribution function (PDF) (right) shows that the probability of inception increases with decreasing time spent at each test condition. Measurements were taken at constant tunnel operating conditions $\left(R e=2.1 \times 10^{6}\right.$ and $\left.\sigma=0.87\right)$. 
the minimum vortex pressure decreases with increasing incidence, and more nuclei become susceptible to cavitation. Of greater interest is the high degree of scatter, particularly for incidences of $5.9^{\circ}$ and smaller. This is most likely due to the spatial and size distributions of the nuclei, rather than pressure fluctuations in the vortex core. The concentration of susceptible nuclei decreases with decreasing incidence, as less tension is applied in the vortex core. This results in less frequent cavitation events, resulting in greater scatter in the inception data. Unsteady pressure fluctuations in the vortex core are not expected to vary significantly across pre-stall incidences and therefore do not appear to contribute as much to the observed scatter.

Vortex strength decreases with decreasing incidence, leaving only weaker nuclei susceptible to cavitation, which are present in lower concentrations and thus sparsely distributed in the flow. The probability of capture and activation of a susceptible nucleus is reduced, resulting in times until inception of up to $240 \mathrm{~s}$ (at $5.51^{\circ}$ ). It should be noted that a threshold incidence exists, below which inception is not physically possible as the vortex does not have sufficient strength to activate even the weakest nucleus. This presumably occurs when the minimum pressure in the vortex core, $p_{\min }$, is higher than the vapour pressure.

A probability density function (PDF) is also provided in Fig. 5 for the time until inception at $6.0^{\circ}$. Measurements were repeated 455 times at this condition to quantify the probabilistic nature of TVC inception. Inception is most likely to occur within $0-20 \mathrm{~s}$ than in any other time interval. However, times until inception of over $300 \mathrm{~s}$ were also observed. These occurrences were rare, but are again indicative of the probabilistic nature of the tensile strength of a body of water and its effect on TVC behaviour.

Some consideration should be given as to how these results would differ for a nuclei abundant flow. With higher concentrations of susceptible nuclei, the time until inception is expected to approach zero across all incidences at and above the threshold incidence $\left(p_{\min }<p_{\mathrm{v}}\right)$, as opportunities for capture and activation become more frequent. The scatter in the data would disappear as a consequence. In this way, smaller sample sizes would be required to accurately characterise TVC inception behaviour as the concentration of susceptible nuclei increases.

Likewise at higher Reynolds numbers, the time until inception is also expected to approach zero across all incidences at and above the threshold incidence. A thinner boundary layer results in a smaller vortex core radius and hence lower minimum pressure for a constant circulation (McCormick, 1962; Franc and Michel,
2006). This results in a higher concentration of susceptible nuclei in the flow, a higher probability of capture and thus shorter times until inception.

The dependence of the incipient cavitation number on the spatial distribution of susceptible nuclei was not limited to the test approach, inception definition and operating conditions presented in Fig. 5. The incipient and desinent incidences are given in Fig. 6 for 58 different cavitation numbers. The effects of Reynolds number and relative saturation level of dissolved oxygen in the plenum are also provided. The incidence was continuously increased until TVC inception was detected, then continuously decreased until TVC desinence occurred. Inception is defined here as when a persistent cavity overlaps with the hydrofoil planform, when viewed from the side of the test section. Desinence is defined by the disappearance of the cavity from the test section.

The inception and desinence numbers trend with incidence due to pressure reduction in the vortex as the lift increases. The inception results exhibit similar scatter as presented earlier in Fig. 5, again due to variations in the time until inception of a susceptible nucleus. Meanwhile, the desinence data exhibit less variability and a linear trend can be observed between the desinent cavitation number and incidence for cavitation indices less than three. The lower scatter suggests that the random spatial distribution of the natural nuclei population does not play a significant role in TVC desinence. The pressure in the vortex core relative to vapour pressure is likely to control the disappearance of a tip vortex cavity, and the desinence data appear to represent the conditions at which the minimum vortex core pressure increases above vapour pressure. Experimental measurements or numerical simulations of the vortex core pressure at these conditions may be used to confirm this hypothesis.

Supplementary tests were performed by measuring the inception angle and spending $60 \mathrm{~s}$ at each $0.1^{\circ}$ interval (triangles in Fig. 6). This enabled an assessment of how the inception angle is affected by exposure of a larger volume of water to the low-pressure vortex core. In general, smaller inception angles were measured, approaching the previously measured desinence angles. This is not surprising, as a larger volume of water passes through the streamtube of opportunity (i.e. the low pressure volume in which nucleus activation is possible), which increases the probability of nuclei capture and activation. The scatter in the inception data decreases for the longer time spent at each test condition, indicating that the incipient cavitation number, when determined in this manner, is less sensitive to the random spatial distribution of nuclei in the flow. Given the results in Fig. 5, which show that cavitation incep- 


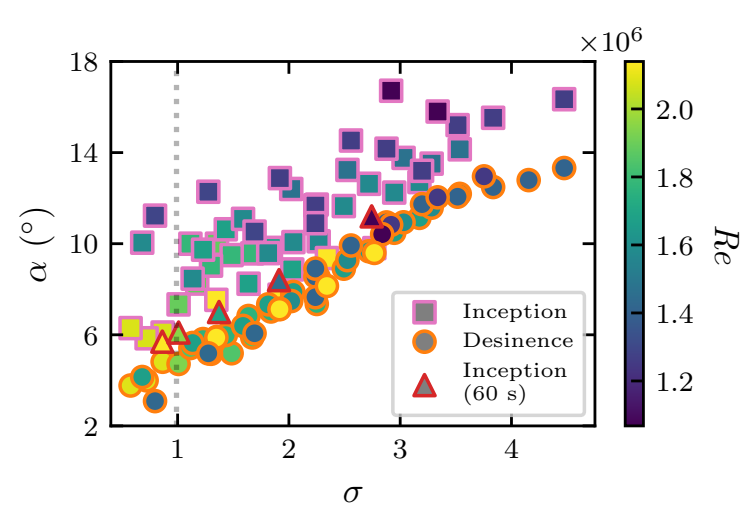

(a) Effect of Reynolds number, Re

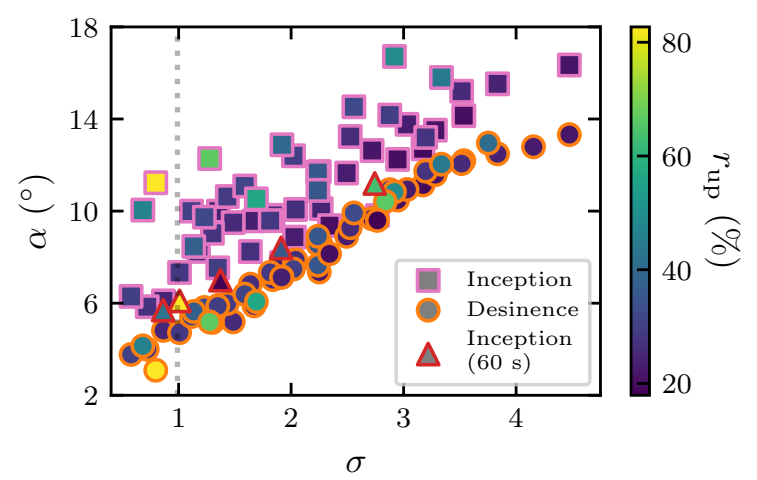

(b) Effect of relative saturation of dissolved oxygen in the plenum, $r_{\text {up }}$

Fig. 6 Variation of inception and desinence angles, $\alpha$, with cavitation number, $\sigma$. Inception is defined as when a persistent cavity overlaps with the hydrofoil planform. Greater variability is observed in the measured inception angles than the desinence. This suggests that the randomness of natural nuclei capture in vortical flows plays a greater role in the inception process. By spending $60 \mathrm{~s}$ at each test condition, lower inception angles were generally measured for a given cavitation number. Reynolds numbers at the upper end of the range tested $\left(1.1 \times 10^{6}-2.1 \times 10^{6}\right)$ tend to correspond to lower cavitation numbers. The relative saturation of dissolved oxygen does not significantly affect the results across the range tested, however, additional data are required to confirm this. The vertical line corresponds to the conditions of Fig. 7 .

tion may not occur for over $300 \mathrm{~s}$, a further increase in the time spent at each test condition should reduce the inception angles further.

Regardless of the time spent at each test condition, the desinence angles provide a lower bound on the inception angles, i.e. a desinence angle is always smaller than its corresponding inception angle. These angles would be expected to be the same if vapour pressure was the only controlling factor. This hysteretical nature has been recently investigated by Amini et al. (2019). An increase in the desinent cavitation index was observed with increasing dissolved gas content, due to diffusion of gas into the cavity in the presence of a laminar separation bubble which protects the vortex core. Arndt et al. (1991) attributed gaseous diffusion into the laminar separation zone to long residence times, while Green (1988) proposed three possible explanations for the hysteretical behaviour:

1. the recirculation of nuclei generated during the cavitation process,

2. the propagation of pressure waves from collapsing bubbles resulting in the activation of unstable nuclei, and

3. the effect of strong cavitation on the underlying single phase flow which could increase the chances of further cavitation.

It may also be due to differences in the energy required to move from one state to another (i.e. condensation during the desinence process as opposed to vaporisation during inception). The observation of this lower bound may also have implications for the cavitation characterisation of components from a practical perspective. That is, the desinence values could be used as a conservative predictor of the inception threshold, as noted by Chang et al. (2009).

The data in Fig. 6 were taken across a range of Reynolds numbers. It is shown in Fig. 6(a) that higher Reynolds numbers tend to correspond to lower cavitation numbers, as the two non-dimensional parameters vary inversely with flow velocity. As explained earlier, tip vortex strength is expected to increase with increasing Reynolds number, resulting in cavitation inception at lower incidences or higher cavitation numbers. This is indeed the case for the 'Inception' data. Furthermore, an increase in flow velocity increases the rate of nuclei capture by the tip vortex, which could also result in lower inception incidences or higher inception indices.

In Fig. 6(b), inception data appear to be unaffected by the relative saturation of dissolved oxygen in the plenum, which would suggest that the nuclei population did not change, as found by Khoo et al. (2020). While dissolved gas and diffusion effects are known to influence desinent (Amini et al., 2019) and general (Holl, 1960; Arndt et al., 1991) cavitation behaviour, this was not the primary focus of the present study and there are limited data points upon which to draw conclusions. This area warrants further research.

A histogram of inception and desinence angle measurements, as detected by the photodiode, repeated 72 times at constant Reynolds and cavitation numbers is provided in Fig. 7. The incidence was continuously increased until TVC inception was detected, then continuously decreased until TVC desinence occurred. This is equivalent to repeated measurements at $\sigma=0.99$ in Fig. 6, at a fixed Reynolds number. 


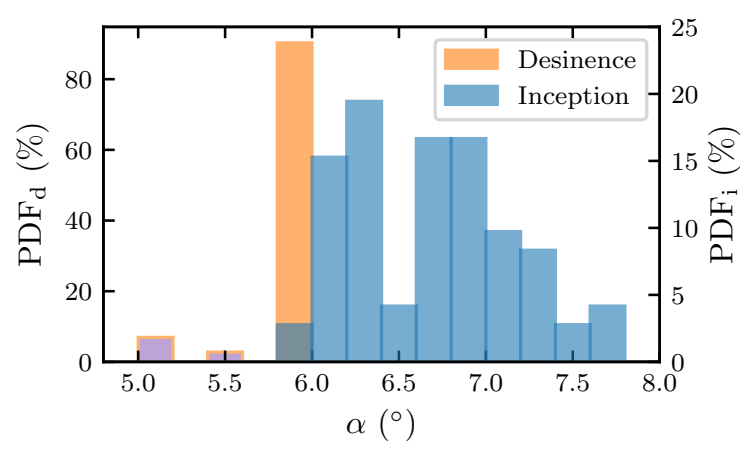

Fig. 7 Histograms of desinent (orange, left axis) and incipient (blue, right axis) cavitation angles. Inception is detected when a cavity breaks a laser beam about 0.15 chord lengths downstream of the tip. Hydrofoil incidence, $\alpha$, was continuously increased from $5.0^{\circ}$ until inception, then continuously decreased until desinence. The measured inception angles exhibit greater variability than desinence angles, for which several outliers can be observed at lower incidences (purple). Measurements were taken at constant tunnel operating conditions $\left(R e=1.7 \times 10^{6}\right.$ and $\left.\sigma=0.99\right)$, which corresponds to the vertical line in Fig. 6.

The lower scatter in the desinence data relative to the inception data is consistent with observations in Fig. 6. All of the desinence data lie within the $5.8^{\circ}-6.0^{\circ}$ interval with the exception of several outliers at lower incidences, where cavities sustained even with weaker tip vortices. This behaviour is considered in further detail in Sect. 3.2.

The distribution of the inception data does not yet appear converged. This indicates that a larger sample size (e.g. $\mathcal{O}(100-1000))$ is required to fully describe the statistical distribution of the inception angle in a nuclei deplete flow. Alternatively, an idea of the spread of the distribution could be found with as few as ten observations.

It is commonly known that Reynolds number and lift coefficient affect vortex strength and thus tip vortex cavitation inception behaviour. Furthermore, the tensile strength of a body of water determines how much tension the liquid can sustain prior to rupture. The results presented thus far demonstrate that the effective tensile strength is probabilistic in nature, and is influenced by test methodology which controls the volume of water, and hence number of nuclei, exposed to low pressures. This volume changes with not only the freestream velocity and the model scale, but also the time spent at each test condition and the increments in the variables of interest. This in turn affects the measured incipient cavitation number. When conducting TVC inception measurements for practical applications, consideration should be given to the relevant time scales and suitable waiting times should be employed. The observation that the inception angle tends to increase with decreasing exposure time may have implications for cavitation inception management for practical applications.

\subsection{Cavitation desinence hysteresis}

In order to explain the existence of the outlying desinence data in Fig. 7, the same data set was plotted as desinence angle, $\alpha_{\mathrm{d}}$, against inception angle, $\alpha_{\mathrm{i}}$ (Fig. 8). Two main modes of behaviour are evident. One colour represents desinence angles that remain relatively constant when the inception angle is $7.3^{\circ}$ or smaller. For the other, smaller desinence angles are observed when the inception angle is $7.4^{\circ}$ or larger. The data corresponding to the latter mode comprise the outliers in Fig. 7. A transition region lies between $7.3^{\circ}-7.4^{\circ}$ where both behaviours are observed.

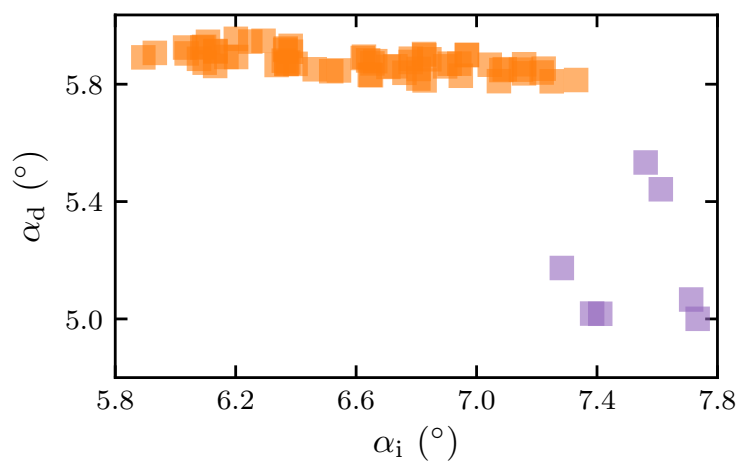

Fig. 8 Desinence angle, $\alpha_{\mathrm{d}}$, as a function of inception angle, $\alpha_{\mathrm{i}}$, using the same data as in Fig. 7. Two modes are evident: large desinence angles correspond to smaller inception angles (orange), while small desinence angles occur for large inception angles (purple). The data points for the latter mode correspond to the outliers in Fig. 7. Measurements were taken at constant tunnel operating conditions $\left(R e=1.7 \times 10^{6}\right.$ and $\sigma=0.99)$.

The differences between the two modes were investigated by taking photographs of cavity topologies at varying stages of development, as shown in Fig. 9. The cavity topology can appear as an unattached tip vortex cavity at smaller incidences, or a cavity attached to the hydrofoil tip at larger incidences. It is evident that topological differences prior to desinence are responsible for the existence of two desinence modes. Similar observations were made in qualitative terms by Green (1988), who stated that the attachment of a trailing vortex cavity to the hydrofoil tip significantly influences the flow field. As a result, a higher cavitation number, sometimes higher than the incipient cavitation number, is required to detach the cavity from the hydrofoil. 


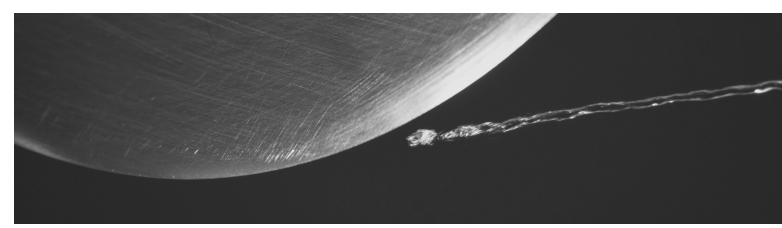

(a) $\alpha=6.8^{\circ}$, Unattached TVC

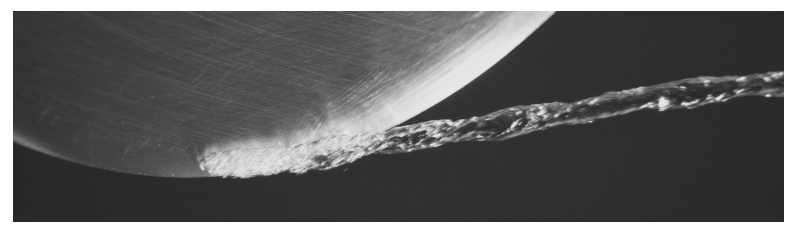

(b) $\alpha=7.9^{\circ}$, Attached TVC

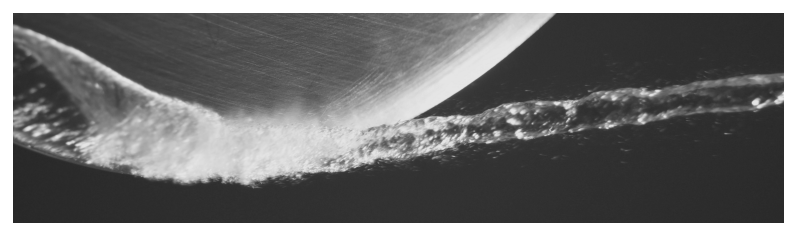

(c) $\alpha=8.1^{\circ}$, Attached TVC (with sheet cavitation)

Fig. 9 Photographs of different stages of tip vortex cavitation (TVC) development. The flow is from left to right. The cavity is sometimes unattached (a) and sometimes attached (b, c). With more tension, leading-edge sheet cavitation appears simultaneously with TVC (c). The change in cavity topology is responsible for the lower desinence angles observed in Figs. 7 and 8 (purple data). Test conditions were $R e=1.9 \times 10^{6}$ and $\sigma=1.4$

A photographic comparison was also conducted for the changing cavitation number approach, as presented in Fig. 10. Two inception-desinence runs are shown. For the first, the cavitation number was lowered well below the incipient value to a minimum value of $\sigma_{\min }=1.31$, with the appearance of simultaneous leading-edge sheet and tip vortex cavitation. The consequent desinent cavitation number of 2.32 for the desinence of attached cavitation is significantly higher than the 1.94 observed for the second run (i.e. desinence of unattached cavitation) where only an unattached tip vortex cavity was observed at the minimum cavitation number of 1.85 . This hysteretical observation further supports the theory that cavity topology at the minimum cavitation number affects TVC desinence behaviour. Gaseous diffusion is known to delay the disappearance of tip vortex cavities at higher cavitation numbers (Amini et al., 2019). Multiple observations of a tip vortex cavity in the 'attached' state without a sheet cavity (Fig. 9(b)), prior to desinence, suggest that cavity attachment affects or controls desinence behaviour.

Stable, attached cavities are often associated with laminar separation bubbles which can stabilise the local flow and shelter the tip vortex core (Amini et al., 2019). Laberteaux and Ceccio (2001) observed the steady closure of a vapour-filled cavity due to an oblique re-entrant jet. Adhesive forces due to interfacial phenomena at the gas-solid-liquid interface may also play a role in desinence hysteresis behaviour (Danov et al., 2016). This raises an interesting question about the influence of material and surface finish on cavity detachment behaviour. Alternatively, this phenomenon may be related to the supply of water vapour from the leading-edge cavity into the vortex.

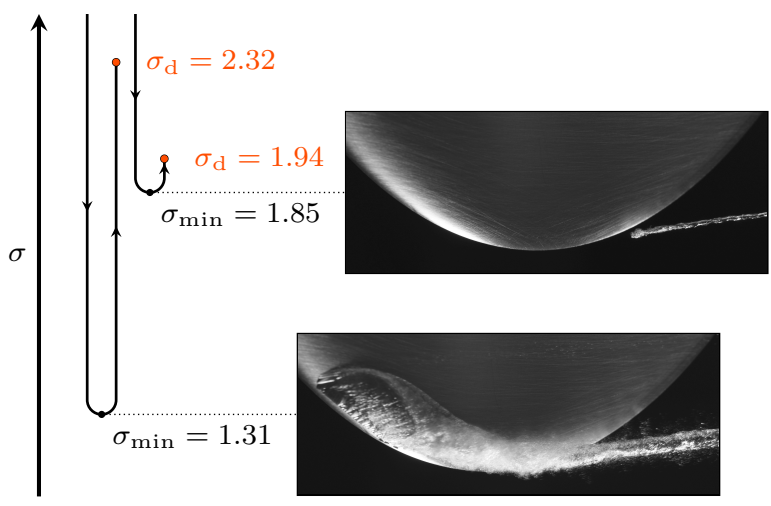

Fig. 10 Schematic of different cavitation desinence tests for identical tunnel conditions $\left(\alpha=8.0^{\circ}\right.$ and $\left.R e=1.7 \times 10^{6}\right)$. Different topologies (as shown in photographs) were generated by reducing the cavitation number to two different minimum values $\left(\sigma_{\min }\right)$. The desinent cavitation number, $\sigma_{\mathrm{d}}$, is dependent on the cavity topology.

The effect of the different cavity topologies at the minimum cavitation number on the relationship between desinent cavitation number and incidence was investigated by varying the cavitation number at fixed incidences for a constant Reynolds number (Fig. 11). The test section pressure was reduced in $5 \mathrm{kPa}$ steps from a non-cavitating condition, with $60 \mathrm{~s}$ spent at each test condition to monitor for inception. This was repeated until TVC inception was detected visually, then either:

1. the test section pressure was continuously increased until TVC desinence occurred, or

2. the test section pressure was decreased further until a leading-edge sheet cavity appeared then continually increased until desinence.

The minimum cavitation numbers reached during the tests are indicated by the circles, and the colour corresponds to the cavity state at this minimum cavitation number. The 'attached' condition required a lower cavitation number. A total of 38 tests were carried out, at 21 unique incidences between $3.5^{\circ}$ and $10.0^{\circ}$.

Below $4.9^{\circ}$, the cavity always manifests as simultaneous leading-edge sheet and tip vortex cavitation, i.e. an isolated tip vortex cavity is not observed. The desinent cavitation number is linearly dependent on the 
incidence, with a slope of $0.17 /^{\circ}$, and is indicated by the dotted line. In this regime, the minimum pressure in the flow is at the leading-edge of the hydrofoil.

For $\alpha \geq 4.9^{\circ}$, different desinence modes are again evident. The unattached mode, shown in orange, is linear across the range of incidences with a slope of $0.42 /^{\circ}$. The minimum cavitation numbers required to induce this mode are close to the desinent values. That is, only a small hysteresis exists between the incipient and desinent cavitation numbers for the desinence of unattached cavitation. This may be because the cavity is not sheltered by the stabilised flow about the tip, or no additional energy is required to detach the cavity from the hydrofoil to overcome interfacial phenomena. The mode with higher desinent cavitation numbers, which corresponds to cavity attachment, has a steeper slope of $0.53 /{ }^{\circ}$ for $\alpha \geq 5.5^{\circ}$. The cavitation number hysteresis is much larger for the desinence of attached cavitation, as observed in Fig. 11. Below $\alpha=5.5^{\circ}$, the attached mode manifests with a lower slope, which appears to be an extension of the regime for which simultaneous leadingedge sheet and tip vortex cavitation occur at inception (dotted line). This suggests that desinence values here may correspond to vapour pressure at the leading edge.

The desinent cavitation numbers for the unattached regime show excellent linearity $\left(R^{2}=0.999\right)$ with incidence. This supports the findings of Fruman and Dugue (1994), but others have observed different relationships (e.g. 'nearly linearly' by McCormick (1962), lift coef- ficient exponents of 1.4 and 2 by Arndt and Keller (1992)). The desinence data series for the attached regime may be interpreted as a polynomial with an index higher than 1 due to the 'kink' at 5.5', which could explain the higher indices noted in other work. Additionally, if a set of desinence values from mixed cavitation modes was used, a non-linear relationship may be found.

Finally, the close agreement observed between the desinence data in Figs. 6 and 11 suggests that the test dmethodology (i.e. changing incidence or cavitation number) has no significant effect.

\section{Conclusions}

Tip Vortex Cavitation (TVC) behaviour about an elliptical planform hydrofoil has been studied in a nuclei deplete flow. Significant scatter in the inception data indicates that the tensile strength of a body of water is not constant, but rather varies throughout and is thus probabilistic in nature. At a given test condition, a cavity may not incept for a long time if the water is sufficiently deplete of nuclei. However, the volume of water exposed to low pressures in the vortical flow increases with time spent at each test condition, thereby increasing the probability of nuclei capture and activation. For nuclei deplete flows in particular, multiple measurements (e.g. $\mathcal{O}(100-1000))$ are required to accurately quantify the probabilistic nature of TVC in-

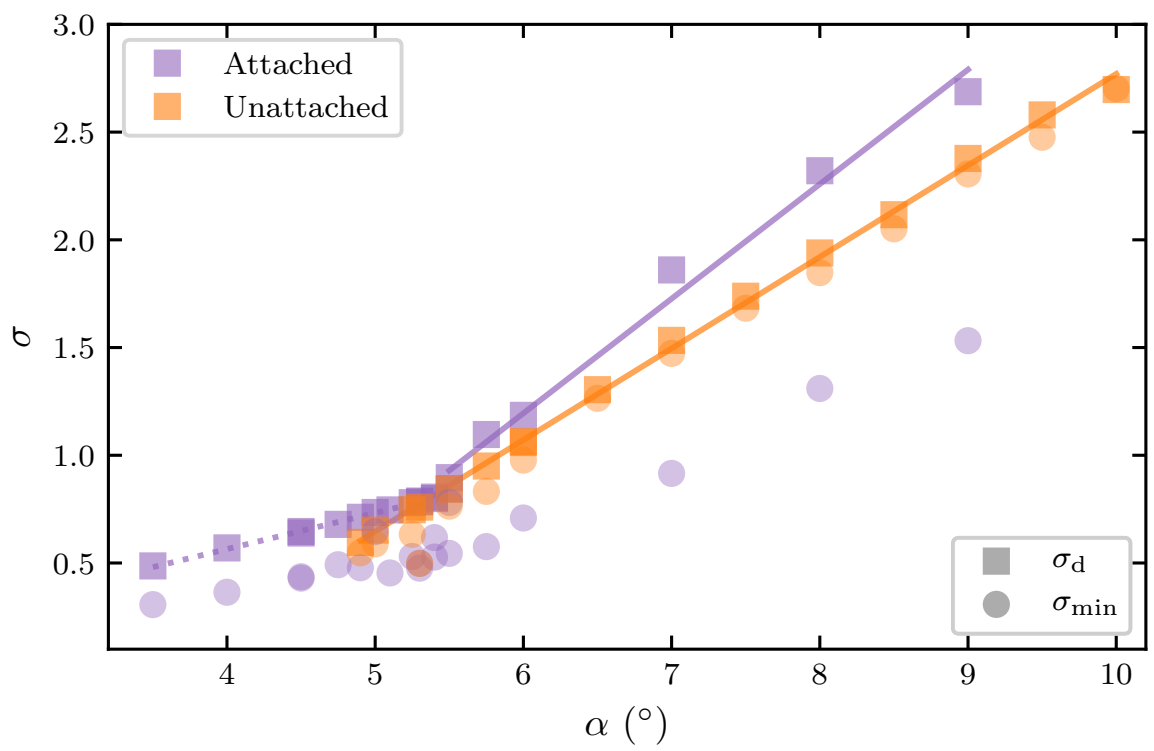

Fig. 11 Variation of desinent cavitation number, $\sigma_{\mathrm{d}}$ in squares, with incidence, $\alpha$, and cavity state (attached in purple and unattached in orange). Above $5.5^{\circ}$, two distinct desinence modes exist according to the topology. The mode with higher $\sigma_{\mathrm{d}}$ values corresponds to the attached cavity state. Below $5.5^{\circ}$, the influence of the leading-edge pressure distribution is indicated by the change in slope of the data. The minimum cavitation numbers, $\sigma_{\min }$, for the tests are shown as circles and determine whether the cavity attaches to the hydrofoil. The Reynolds number was $1.8 \times 10^{6}$. 
ception. It can be reasoned that a smaller sample size will be required for nuclei abundant flows due to the higher nuclei flux through the low pressure region and increased probability of inception. To confirm this, further tests are required using flows injected with higher concentrations of larger microbubbles compared to the population studied here.

Different TVC desinence behaviours are observed, dependent on the topology of the cavitating flow. For the desinence of attached cavitation, the desinent cavitation number is higher and larger hysteresis is observed compared to the desinence of unattached cavitation. A number of possible reasons for this are proposed, including stabilisation of the cavity flow by a laminar separation bubble, and adhesive forces present at the gas-liquid-solid interface due to interfacial phenomena. Desinence measurements are not affected by nuclei, resulting in lower scatter in the observed cavitation numbers. While the repeatability of desinence simplifies cavitation measurements, inception is often of greater practical interest.

Further work is required to correlate vortex pressure and the tensile strength of a body of water, taking into account factors such as bubble dynamics during capture and unsteady pressure fluctuations in the vortex core. This would assist in the development of a TVC inception prediction model which takes a known nuclei population and pressure field, and computes the probability of inception for a given operating condition.

Acknowledgements The authors acknowledge the support of the University of Tasmania and the Defence Science and Technology Group. The authors thank AMC technical officers, Mr Robert Wrigley and Mr Steven Kent for providing technical assistance with test facility configuration and operation.

\section{References}

Amini A, Reclari M, Sano T, Iino M, Dreyer M, Farhat M (2019) On the physical mechanism of tip vortex cavitation hysteresis. Exp Fluids 60(7):118

Amromin E (2006) Two-range scaling for tip vortex cavitation inception. Ocean Eng 33(3-4):530-534

Arndt R, Dugue C (1992) Recent advances in tip vortex cavitation research. In: International symposium on propulsors and cavitation, pp 142-149

Arndt R, Maines B (2000) Nucleation and bubble dynamics in vortical flows. J Fluids Eng, Trans ASME 122(3):488-493, DOI 10.1115/1.1286994

Arndt R, Arakeri V, Higuchi H (1991) Some observations of tip-vortex cavitation. J Fluid Mech 229:269289
Arndt RE, Keller AP (1992) Water quality effects on cavitation inception in a trailing vortex. J Fluids Eng, Trans ASME 114(3):430-438

Arndt RE, Maines BH (1994) Further studies of tip vortex cavitation. In: 2nd international symposium on cavitation, Tokyo

Baker G, Barker S, Bofah K, Saffman P (1974) Laser anemometer measurements of trailing vortices in water. J Fluid Mech 65(2):325-336

Billet M, Holl J (1979) Scale effects on viscous types of limited cavitation. In: International symposium on cavitation inception, pp 2-7

Blake Jr F (1949) The tensile strength of liquids: A review of the literature. Technical memo no. 9. Tech. rep., Acoustic Research Lab., Harvard Univ.

Brandner P (2018) Microbubbles and cavitation: Microscales to macroscales. In: 10th international symposium on cavitation

Brandner P, Lecoffre Y, Walker G (2007) Design considerations in the development of a modern cavitation tunnel. In: 16th Australasian fluid mechanics conference

Briançon-Marjollet L, Merle L (1996) Inception, development and noise of a tip vortex cavitation. In: $21 \mathrm{st}$ symposium on naval hydrodynamics, pp 851-864

Ceccio SL, Brennen CE (1991) Observations of the dynamics and acoustics of travelling bubble cavitation. J Fluid Mech 233:633-660

Chang N, Yakushiji R, Ganesh H, Ceccio S (2009) Mechanism and scalability of tip vortex cavitation suppression by water and polymer injection. In: 7th international symposium on cavitation, 149, pp 1-12

Chen L, Zhang L, Peng X, Shao X (2019) Influence of water quality on the tip vortex cavitation inception. Phys Fluids 31(2):023303

Danov KD, Stanimirova RD, Kralchevsky PA, Marinova KG, Stoyanov SD, Blijdenstein TB, Cox AR, Pelan EG (2016) Adhesion of bubbles and drops to solid surfaces, and anisotropic surface tensions studied by capillary meniscus dynamometry. Adv Colloid Interface Sci 233:223-239

Franc JP, Michel JM (2006) Fundamentals of Cavitation. Springer Science \& Business Media

Franklin R (1992) A note on the radius distribution function for microbubbles of gas in water. In: ASME Cavitat Multiphase Flow Forum, FED-Vol., vol 135, pp 77-85

Fruman D, Dugue C (1994) Tip vortex roll-up and cavitation. In: 19th symposium on naval hydrodynamics, pp 633-654

Gavrilov L (1969) On the size distribution of gas bubbles in water. Sov Phys - Acoustics 15(1):22-24 
Gindroz B (1995) Propeller cavitation characteristics: The practical interest of nuclei measurements in test facilities and at sea. In: Proceedings of ASME FED symposium on cavitation

Gindroz B, Billet M (1998) Influence of the nuclei on the cavitation inception for different types of cavitation on ship propellers. J Fluids Eng 120(1):171-178

Green S (1988) Tip vortices: Single phase and cavitating flow phenomena. $\mathrm{PhD}$ thesis, California Institute of Technology

Green S (1991) Correlating single phase flow measurements with observations of trailing vortex cavitation. J Fluids Eng 113(1):125-129

Holl J (1960) An effect of air content on the occurrence of cavitation. J Basic Eng pp 941-946

Hsiao C, Chahine G (2008) Scaling of tip vortex cavitation inception for a marine open propeller. In: 27th symposium on naval hydrodynamics, Seoul, Korea, pp 5-10

Hsiao CT, Chahine GL (2005) Scaling of tip vortex cavitation inception noise with a bubble dynamics model accounting for nuclei size distribution. J Fluids Eng 127(1):55-65

Khoo M, Venning J, Pearce B, Brandner P, Lecoffre Y (2016) Development of a cavitation susceptibility meter for nuclei size distribution measurements. In: 20th Australasian fluid mechanics conference

Khoo M, Venning J, Pearce B, Takahashi K, Mori T, Brandner P (2020) Natural nuclei population dynamics in cavitation tunnels. Exp Fluids 61(2):1-20

Laberteaux K, Ceccio S (2001) Partial cavity flows. part 2 . cavities forming on test objects with spanwise variation. J Fluid Mech 431:43-63

Lecoffre Y (1999) Cavitation Bubble Trackers. A. A. Balkema

McCormick B (1962) On cavitation produced by a vortex trailing from a lifting surface. J Basic Eng 84(3):369-378

Messino D, Sette D, Wanderlingh F (1963) Statistical approach to ultrasonic cavitation. J Acoust Soc Am 35(10):1575-1583

Meyer R, Billet M, Holl J (1992) Freestream nuclei and traveling-bubble cavitation. J Fluids Eng 114(4):672679

Mørch KA (2000) Cavitation nuclei and bubble formationa dynamic liquid-solid interface problem. J Fluids
Eng 122(3):494-498

O'Hern T, d'Agostino L, Acosta A (1988) Comparison of holographic and Coulter counter measurements of cavitation nuclei in the ocean. J Fluids Eng 110(2):200-207

Oweis G, van der Hout I, Iyer C, Tryggvason G, Ceccio S (2005) Capture and inception of bubbles near line vortices. Phys Fluids 17(2)

Pennings P, Westerweel J, Van Terwisga T (2015) Flow field measurement around vortex cavitation. Exp Fluids 56(11):206

Randolph K, Dierssen HM, Twardowski M, CifuentesLorenzen A, Zappa CJ (2014) Optical measurements of small deeply penetrating bubble populations generated by breaking waves in the Southern Ocean. J Geophys Res: Oceans 119(2):757-776

Schiebe FR (1969) The influence of gas nuclei size distribution on transient cavitation near inception. Tech. rep., St. Anthony Falls Laboratory

Shen Y, Gowing S, Pierce R (1984) Cavitation susceptibility measurements by a venturi. In: ASME international symposium on cavitation inception

Shen YT, Jessup S, Gowing S (2009) Tip vortex cavitation inception scaling for high reynolds number application. In: ASME/JSME 2003 4th joint fluids engineering summer conference, American Society of Mechanical Engineers Digital Collection, pp 233-239

Sinding KM, Hanson DR, Krane MH, Koncoski J (2018) Insight into tip vortex cavitation using velocity field measurements. In: 10th international symposium on cavitation, ASME Press

Song M, Xu L, Peng X, Tang D (2017) An acoustic approach to determine tip vortex cavitation inception for an elliptical hydrofoil considering nuclei-seeding. Int J Multiph Flow 90:79-87

Venning J, Khoo M, Pearce B, Brandner P (2018) Background nuclei measurements and implications for cavitation inception in hydrodynamic test facilities. Exp Fluids 59(4):71

Yakushiji R (2009) Mechanism of tip vortex cavitation suppression by polymer and water injection. $\mathrm{PhD}$ thesis, The University of Michigan

Zhang LX, Chen LY, Peng XX, Shao XM (2017) The effect of water quality on tip vortex cavitation inception. J Hydrodynamics 29(6):954-961 